\title{
Effectiveness of Paid Search Advertising: Experimental Evidence
}

\section{Citation}

Dai, Weijia (Daisy), and Michael Luca. "Effectiveness of Paid Search Advertising: Experimental Evidence." Harvard Business School Working Paper, No. 17-025, October 2016.

\section{Permanent link}

http://nrs.harvard.edu/urn-3:HUL.InstRepos:30013618

\section{Terms of Use}

This article was downloaded from Harvard University's DASH repository, and is made available under the terms and conditions applicable to Open Access Policy Articles, as set forth at http:// nrs.harvard.edu/urn-3:HUL.InstRepos:dash.current.terms-of-use\#OAP

\section{Share Your Story}

The Harvard community has made this article openly available.

Please share how this access benefits you. Submit a story.

\section{Accessibility}




\section{Effectiveness of Paid Search} Advertising: Experimental Evidence

Weijia (Daisy) Dai Michael Luca

Working Paper 17-025 


\title{
Effectiveness of Paid Search Advertising: Experimental Evidence
}

\author{
Weijia (Daisy) Dai
}

Lehigh University

Michael Luca

Harvard Business School

Working Paper 17-025 


\title{
Effectiveness of Paid Search Advertising: Experimental Evidence*
}

\author{
Weijia (Daisy) Dai \\ Lehigh University
}

\author{
Michael Luca \\ Harvard Business School
}

October 1, 2016

\begin{abstract}
Paid search has become an increasingly common form of advertising, comprising about half of all online advertising expenditures. To shed light on the effectiveness of paid search, we design and analyze a large-scale field experiment on the review platform Yelp.com. The experiment consists of roughly 18,000 restaurants and 24 million advertising exposures - randomly assigning paid search advertising packages to more than 7,000 restaurants for a three-month period, with randomization done at the restaurant level to assess the overall impact of advertisements. We find that advertising increases a restaurant's Yelp page views by $25 \%$ on average. Advertising also increases the number of purchase intentions - including getting directions, browsing the restaurant's website, and calling the restaurant - by 18\%, 9\%, and 13\% respectively, and raises the number of reviews by $5 \%$, suggesting that advertising also affects the number of restaurantgoers. All advertising effects drop to zero immediately after the advertising period. A back of the envelope calculation suggests that advertising would produce a positive return on average for restaurants in our sample.
\end{abstract}

\footnotetext{
${ }^{*}$ We thank Susan Athey, Garrett Johnson, Randall Lewis and participants at the NBER Summer Institute for valuable comments. We thank Yelp, and especially Geoff Donaker, Matt Halprin, Luther Lowe, Travis Brooks, Brian Dean, and Stephen Lyons for providing support for this experiment. Please contact Daisy Dai (dai@lehigh.edu) or Mike Luca (mluca@hbs.edu) for correspondence.
} 


\section{Introduction}

Internet advertising has been the fastest-growing marketing channel in recent years, accounting for roughly $\$ 60$ billion of spending in the United States alone in 2015. The rise of digital advertising has been dramatic - more than doubling over the past five years alone. Paid search, in which advertisements are placed alongside search results, comprises the largest share of online advertising expenditures.

In the offline world, advertising has historically resembled a credence good, where the effectiveness of a product is taken largely on faith. Even after an advertising campaign is implemented, limited access to outcome data and exogenous variation in exposure have often prevented credible estimates of the impact of advertising.

In principle, the digital age provides new opportunities to evaluate the effectiveness of advertisements, enabled by granular data about users and increased feasibility of experimentation. Yet estimating the effectiveness of digital advertising remains challenging. Correlations in observational data can yield biased estimates because advertisements are more likely to be shown to people who are interested and therefore prone to make a purchase even without an advertisement (Johnson, Lewis and Reilly, 2014; Blake et al. 2015; Gordon et al. 2016). Moreover, large sample sizes are needed to estimate what are often modest impacts of individual advertising strategies with sufficient precision (Lewis and Reiley 2014; Lewis and Rao 2015; Athey and Imbens 2016).

To explore the impact of paid search advertising across a broad set of small businesses, we design and analyze a large-scale field experiment on the popular review website Yelp.com. On Yelp, a business can purchase standard advertising packages for a fixed rate, which guarantees a minimum number of advertising impressions to be shown each month. Taking these packages as given, we experimentally assign standard advertising packages to more than 7,000 restaurants during a threemonth period, sending out roughly 24 million total advertising exposures. We focus on restaurants that had not actively advertised on Yelp prior to the experiment. We then monitor businesslevel outcomes including page views of the the business's Yelp page (a standard measurement of advertising effectiveness) as well as three measures that are designed to measure customer intentions to go to the restaurant - requests for directions, phone calls to the restaurant from Yelp's mobile page or mobile app, and clicks on the restaurant's own URL on its Yelp page.

Overall, we find that advertising increases a restaurant's Yelp page views by $25 \%$. Within the 
industry, the number of clicks on a page is widely considered an indication of consumer preferences. In fact, companies including Yelp, Bing, and Google design advertisements with this objective in mind. Nonetheless, in principle, users might click on a page that is ultimately a mismatch. To reinforce our interpretation of clicks as a proxy for demand, we select a sample size that was large enough to detect effects on our three indicators of purchase intention.

The standard advertising package leads to 10-20\% more purchase intentions. This reinforces our interpretation that Yelp advertisements lead to increases in demand. However, the increase in conversions is smaller than the increase in page visits. This implies that the marginal visitors are different from the average visitor occurring through an organic click.

As a final indicator of demand, we consider the number of reviews that are left during the advertising period. In principle, if more Yelp users are actually going to the restaurant, then one would expect to see an increase in the number of reviews being left. Consistent with this, we find that advertising leads to a $5.4 \%$ increase in the number of reviews left for a restaurant in a given month. However, the effects of advertising drop to zero immediately after the advertising period, suggesting that advertising temporarily raises awareness of businesses that users would otherwise not discover. A back of the envelope calculation suggests that advertising leads to an $8 \%$ increase in revenue, and would produce a positive return, on average, within our sample.

Our findings contribute to the literature on the effectiveness of online advertising. Most online advertising studies have focused on experiments run by a single large company, enabled by the large consumer base that each of these companies has access to. For example, Blake et al. (2015) and Avery et al. (2016) show that digital advertisements (on Bing and Facebook, respectively) have very little effect on demand for eBay and the College Board. In contrast, we show large average effects across a broad swath of potential advertisers on Yelp. These restaurants are much smaller and less well known than eBay or the College Board and hence may benefit more from advertisements. More generally, our results demonstrate the potential of sponsored search to drive outcomes - even among businesses that have opted not to advertise. Whereas Blake et al. (2015) conclude that many well-branded advertisers might be better off by not advertising, our results raise the possibility that many less-branded non-advertisers might be better off by advertising. 


\section{Experiment and Data}

The experiment was conducted on the popular review platform Yelp.com. Yelp hosts usergenerated reviews of local businesses around the world and provides search functions in browsers as well as its own mobile app. As of 2015, Yelp had roughly 163 million unique visitors monthly. We focus on the US restaurant industry in our experiment.

The goal of the experiment is to examine the effect of the search advertising package currently provided by Yelp. Businesses pay a fix monthly fee for the advertising package and are guaranteed a minimum number of search appearances. Yelp manages and automates the paid search bidding for the advertised businesses. Figure 1 shows examples of paid search appearances.

To evaluate advertising effectiveness, we randomly select businesses to be given advertising packages for three months from August 1, 2015, to October 31, 2015. In choosing the sample, we only include businesses that are eligible to purchase search advertising and are actively operating businesses with basic verified listing information, including photos and a minimum number of trusted reviews with good ratings.

We use stratified sampling in the randomization process. Stratified randomization that randomizes treatment within groups of businesses with similar characteristics increases statistical power (Athey and Imbens 2016; Imbens and Rubin 2015). The strata are described in the following. The first stratum includes Yelp Reservations restaurants. Yelp Reservations is an online reservation system hosted by Yelp that allows consumers to directly make reservations using the simple booking tool on the restaurant's Yelp page. The second stratum includes restaurants that are clients of OpenTable, an Internet booking platform external to Yelp. We use a separate stratum for these restaurants since they are similar and are usually mid-range to high-end full-service restaurants. The third stratum includes restaurants that partner with Yelp's delivery service, EAT24. The fourth stratum includes restaurants in Washington State (because we have done prior research focusing on this state) that do not use OpenTable and are not Yelp partners. These restaurants have less online prominence on average. As shown in Panel B of Figure 3, stratum 4 has fewer reviews than restaurants in other strata. The number of treated restaurants in each stratum is shown in Table 1. We also restrict the proportion of restaurants treated in the Yelp-defined marketing area to be smaller than 10\%. In principle, we can observe two additional metrics beyond the ones in this analysis: reservations for Yelp Reservations restaurants and deliveries for EAT24 restaurants. However, 
a limited number of restaurants use each of these services, preventing us from obtaining conclusive estimates on those two variables.

As shown in Table 1, the final experiment sample includes 18,295, and 7,210 restaurants are given free advertising packages. The experiment started on August 1, 2015, and ended on October 31, 2015. To avoid Hawthorne effects, restaurants are not told about the advertising packages and are removed from the advertising sales lists.

Randomization is done at the business level. We randomize at the business, rather than user, level for two reasons. First, users who are randomly exposed to ads may generate purchases and subsequently reviews (which we did observe in the results), and therefore might influence consumers who are not exposed to ads, generating spillover. Second, we were interested in the overall impact of the standard advertising package on outcomes for a business.

The key metric we use to assess advertising effectiveness is the number of page views of a restaurant's Yelp page, a standard measure in the advertising industry. In addition, we collect conversion metrics to measure consumers' purchase intent - map queries on Yelp, calls following consumers' clicks on a restaurant's phone number on Yelp's mobile page or app, and clicks on a restaurant's own website link on its Yelp page. As an indicator of consumer demand, we also consider reviews left during the advertising period. Finally, for Yelp Reservations restaurants, we observe the number of reservations made through Yelp. However, operational limitations within the company prevented us from having a large enough sample to detect meaningful effects on reservations (which we determined going into the experiment, but could not change).

One advantage of our experiment is that we are able to randomize among a large set of representative restaurants in the market and hence obtain the average treatment effect for businesses typically unable to run their own experiments, and allowing a contrast to some of the large experiments run by individual advertisers. While we present unweighted results, we can reweight to obtain treatment effects for different populations.

\section{Conceptual Framework}

We estimate the average treatment effect using the specification

$$
y_{i t}=\beta_{1} \text { AdsOn }_{i t}+\beta_{2} \text { TreatedPostExpr } i t+\mu_{i}+\delta_{t}+\epsilon_{i t}
$$


where $i$ is a restaurant and $t$ is a month. We sample the periods between January 2015 and December 2015. $A d s O n_{i t}$ is a dummy variable that equals to one for a treated restaurant during the experimental period (8/1/2015-10/31/2015). TreatedPostExpr $i$ is an indicator variable that equals one for a treated restaurant during the post-experiment period $(11 / 1 / 2015-12 / 31 / 2015)$. In the baseline specification, we also control for business and month fixed effects. The parameters of interest are $\beta_{1}$ and $\beta_{2}$, which represent the average treatment effects during and after the experiment (advertising) period.

All results are reported in percent changes to protect level variables that are sensitive within the industry. More specifically, we first calculate the percentage as the ratio of the advertising effect estimated in equation 1 and the control (non-advertising) group average during the treatment period within each strata, and then we take the weighted average across strata. ${ }^{1}$ To calculate the statistical inference for the average percentage gain, we need to note the correlation between treatment effects and control group outcomes in the ratio calculation as well as the correlation of ratios across different strata. We derive the confidence interval of the ratio by bootstrapping. Significance are qualitatively unchanged for level estimates.

We check other choices of specifications for robustness. Using the linear regression framework, we drop the business level fixed effect $\mu_{i}$ and replace it simply by the treatment indicator, and the results are unaffected. We also run the generalized linear regression with Poisson link and we get similar results. For alternative hypothesis testing methods of advertising effects, we run the Fisher exact test to confirm that we can reject the null at each equation level (Athey and Imbens 2016, Young 2016).

We can also view outcomes as resulting from different depths of search, as we expect the advertising effect on consumer activity to decline with each additional step in the search process. For example, the consumer can only click on a restaurant's own website link after visiting the restaurant's Yelp page. Hence, the probability of observing a visit to a restaurant's website can be written as $P(U R L C l i c k)=P($ URLClick $\mid$ PageView $) \times P($ PageView $\mid$ Appearance $) \times P($ Appearance $)$, where appearance is the appearance of the restaurant in the search result. We call the first conditional probability "conversion rate" and the second one "click-through rate." Paid search advertising can increase the number of restaurant URL clicks (i.e. visits to the restaurant's own website) by in-

\footnotetext{
${ }^{1}$ In the baseline results in Table 2, we use the weight as simply the sampling weight across strata.
} 
creasing the chance that the restaurant is seen by consumers (search appearance), but the impact on URL clicks depends on both click-through rate and conversion rate. If the conversion rate for paid clicks is the same as for baseline organic clicks, we should see the same percentage increase in URL clicks and page views. However, the number of URL clicks may increase less if marginal users arriving through advertisements are less likely to click on the URL relative to average consumers viewing the Yelp page.

\section{Results}

The effects of paid search advertising across all outcomes are presented in Table 2, and the effects on key outcomes are plotted in Figure 4. Overall, we find that advertising increases a restaurant's Yelp page views by $24.6 \%$, and the increase is greater for mobile views (30.2\%) than for web browser views $(21.5 \%)$. Across the three conversion metrics, advertising led to an increase in map inquiries by $17.7 \%$, in calls by $12.6 \%$, and in clicks on restaurants' own URLs by $8.8 \%$. This suggests that Yelp advertisements lead to increases in demand.

These results demonstrate that advertisements on Yelp are effective at increasing a variety of metrics indicating consumer intentions to visit the restaurant. We also find that the increase in conversion metrics is smaller than the increase in page views, suggesting that the marginal visitor driven from advertising is less likely than an average visitor to convert to a purchase.

As a final indicator of demand, we examine the number of reviews. We find an average $5.4 \%$ increase in the number of reviews due to advertising. However, while advertising generates more reviews, there is no significant change to the average rating, the review length, or the percent of reviews that are removed by Yelp's filter.

The temporal effect of advertising is shown in the post-experiment estimates column in Table 2 . Overall, we find that the effects of advertising disappear immediately after the advertising period. When estimating advertising effects for the first, second, and third advertising month separately, we do not find any evidence that how long the business has been advertising matters. These can also be seen in Figure 2, which shows the daily total page views for control and treated restaurants in two strata (EAT24 and Washington state restaurants) during our sample period. In this figures, we see that the gain in total page views stays roughly constant across three months, and the gain disappears immediately after the experiment. These findings suggest that advertising provides 
information about a business that users would otherwise not discover.

\section{Discussion}

Overall, our results shed light on the effectiveness of paid search advertising for small businesses. To our knowledge, this is the largest scale advertising effectiveness study in terms of the number of businesses involved. For the types of small businesses that are common on Yelp and similar platforms, we show that advertising does indeed have a large impact. Comparing effects on page views, three consumer intent measures, and the number of reviews, we find lower effects on purchase intent and purchase indicator than on page views. This highlights the fact that the marginal consumers acquired from the paid search advertising are systematically different from the average person viewing the page. While we lack the volume of sales information required to directly estimate the impact of advertising on sales revenue, we obtain sales revenue for a sample of restaurants in Washington state. Looking at the relationship between changes in revenue and changes in page views, we estimate the impact of advertising on sales using this proxy. As we show below, the return of paid search advertising is positive, on average, for our sample of restaurants - which consists of restaurants that were not advertising on Yelp, and one might expect a larger return for businesses that are advertising.

\subsection{The Returns to Advertising}

To estimate the return on advertising, we need an estimate of the dollar value of marginal page views. To do so, we matched a subset of Washington state restaurants (totaling 835 restaurants) back to tax records, which we obtained for the first half of 2015 (the period just before our experiment) from the Washington State Department of Revenue. While we do not have enough data on sales revenue for restaurants in our sample to use this as a dependent variable, we can look at the relationship between changes in sales and changes in page views in the sample, which we use as a proxy for the change in revenue that would be associated with a change in page views generated by advertising. Specifically, we conduct the following regression to obtain the effect of changes in page views on changes in revenue.

$$
\log \left(\text { revenue }_{i t}\right)=\beta \log \left(\text { pageview }_{i t}\right)+\alpha_{i}+\delta_{t}+\epsilon_{t}
$$


where $i$ is the indicator for a restaurant and $t$ the indicator for a quarter. We add restaurant fixed effects to examine within restaurant variations and we add quarterly dummies to control for common time trends. The estimate for $\beta$ is $32.54 \%$ significant at $1 \%$ level with standard error clustered at the business level. This means a $10 \%$ increase in the total number of quarterly page views leads to a $3.3 \%$ increase in quarterly revenue. Advertising leads to an average increase of $24.6 \%$ in total page views according to our experiment, and hence an increase of roughly $8 \%$ in revenue. For example, for a business with $\$ 96,000$ in quarterly sales (the median in Washington state), and given a marginal profit margin for additional sales of roughly around $70 \%,{ }^{2}$ the return on advertising would be $446 \%{ }^{3}$. An important limitation of this calculation is the fact that marginal clicks deriving from advertising may have differential impacts relative to marginal clicks deriving from sales. Nonetheless, this suggests that paid search advertising can be a profitable investment for small businesses, even among ones that are not currently advertising.

\section{References}

[1] Daniel A Ackerberg. Empirically distinguishing informative and prestige effects of advertising. RAND Journal of Economics, pages 316-333, 2001.

[2] Susan Athey and Guido Imbens. The econometrics of randomized experiments. arXiv preprint arXiv:1607.00698, 2016.

[3] Kyle Bagwell. The economic analysis of advertising. Handbook of industrial organization, 3:1701-1844, 2007.

[4] Thomas Blake, Chris Nosko, and Steven Tadelis. Consumer heterogeneity and paid search effectiveness: A large-scale field experiment. Econometrica, 83(1):155-174, 2015.

[5] Tat Y Chan, Chunhua Wu, and Ying Xie. Measuring the lifetime value of customers acquired from google search advertising. Marketing Science, 30(5):837-850, 2011.

[6] J. Cohen. Statistical Power Analysis for the Behavioral Sciences. Taylor \& Francis, 2013.

[7] Benjamin Edelman. The design of online advertising markets. Handbook of Market Design, 2010.

[8] Avi Goldfarb. What is different about online advertising? Review of Industrial Organization, 44(2):115-129, 2014.

\footnotetext{
${ }^{2}$ According to accounting firm Baker Tilly (http://www.bakertilly.com/uploads/restaurant-benchmarking.pdf), the variable cost (food cost, or in other words, the cost of goods sold) is roughly $28-32 \%$ of total sales.

${ }^{3}$ Gain from advertising is $(\$ 96,000 \times 7.97 \% \times 70 \%=) \$ 5356$ per quarter, and the cost of advertising is $\$ 1,200$ per quarter.
} 
[9] Avi Goldfarb and Catherine Tucker. Online display advertising: Targeting and obtrusiveness. Marketing Science, 30(3):389-404, 2011.

[10] Brett Gordon, Florian Zettelmeyer, Neha Bhargava, and Dan Chapsky. A comparison of approaches to advertising measurement: Evidence from big field experiments at facebook. White paper, 2016.

[11] Guido W Imbens and Donald B Rubin. Causal inference in statistics, social, and biomedical sciences. Cambridge University Press, 2015.

[12] Garrett A Johnson, Randall A Lewis, and Elmar I Nubbemeyer. The online display ad effectiveness funnel \& carry-over: A meta-study of ghost ad experiments. Working Paper, 2015.

[13] Garrett A Johnson, Randall A Lewis, and David Reiley. Location, location, location: repetition and proximity increase advertising effectiveness. Available at SSRN 2268215, 2014.

[14] Soohyung Lee and Azeem M. Shaikh. Multiple testing and heterogeneous treatment effects: Re-evaluating the effect of progresa on school enrollment. Journal of Applied Econometrics, 29(4):612-626, 2014.

[15] Randall A Lewis and Justin M Rao. The unfavorable economics of measuring the returns to advertising. The Quarterly Journal of Economics, page qjv023, 2015.

[16] Randall A. Lewis, Justin M. Rao, and David H. Reiley. Here, there, and everywhere: Correlated online behaviors can lead to overestimates of the effects of advertising. In Proceedings of the 20th International Conference on World Wide Web, WWW '11, pages 157-166, New York, NY, USA, 2011. ACM.

[17] Randall A Lewis and David H Reiley. Online ads and offline sales: measuring the effect of retail advertising via a controlled experiment on yahoo! Quantitative Marketing and Economics, 12(3):235-266, 2014.

[18] John A. List, Azeem M. Shaikh, and Yang Xu. Multiple hypothesis testing in experimental economics. Working Paper 21875, National Bureau of Economic Research, January 2016.

[19] Puneet Manchanda, Jean-Pierre Dubé, Khim Yong Goh, and Pradeep K Chintagunta. The effect of banner advertising on internet purchasing. Journal of Marketing Research, 43(1):98$108,2006$.

[20] K.R. Murphy, B. Myors, and A. Wolach. Statistical Power Analysis: A Simple and General Model for Traditional and Modern Hypothesis Tests, Fourth Edition. EBL-Schweitzer. Taylor \& Francis, 2014.

[21] Sridhar Narayanan and Kirthi Kalyanam. Position effects in search advertising: A regression discontinuity approach. Technical report, Tech. rep., Stanford University, 2014.

[22] David H Reiley, Sai-Ming Li, and Randall A Lewis. Northern exposure: A field experiment measuring externalities between search advertisements. In Proceedings of the 11th ACM conference on Electronic commerce, pages 297-304. ACM, 2010.

[23] Oliver J Rutz and Randolph E Bucklin. From generic to branded: A model of spillover in paid search advertising. Journal of Marketing Research, 48(1):87-102, 2011. 
[24] Navdeep S Sahni. Advertising spillovers: Evidence from online field-experiments and implications for returns on advertising. Journal of Marketing Research, 2016.

[25] Navdeep S Sahni and Harikesh Nair. Does advertising serve as a signal? evidence from field experiments in mobile search. Working Paper (January 14, 2016), 2016.

[26] Sha Yang and Anindya Ghose. Analyzing the relationship between organic and sponsored search advertising: Positive, negative, or zero interdependence? Marketing Science, 29(4):602-623, 2010 .

[27] Song Yao and Carl F Mela. A dynamic model of sponsored search advertising. Marketing Science, 30(3):447-468, 2011.

[28] Alwyn Young. Channeling fisher: Randomization tests and the statistical insignificance of seemingly significant experimental results. Technical report, Technical Report, Working paper, 2015 . 


\section{Figures and Tables}

Figure 1: Snapshot of a Paid Search Result

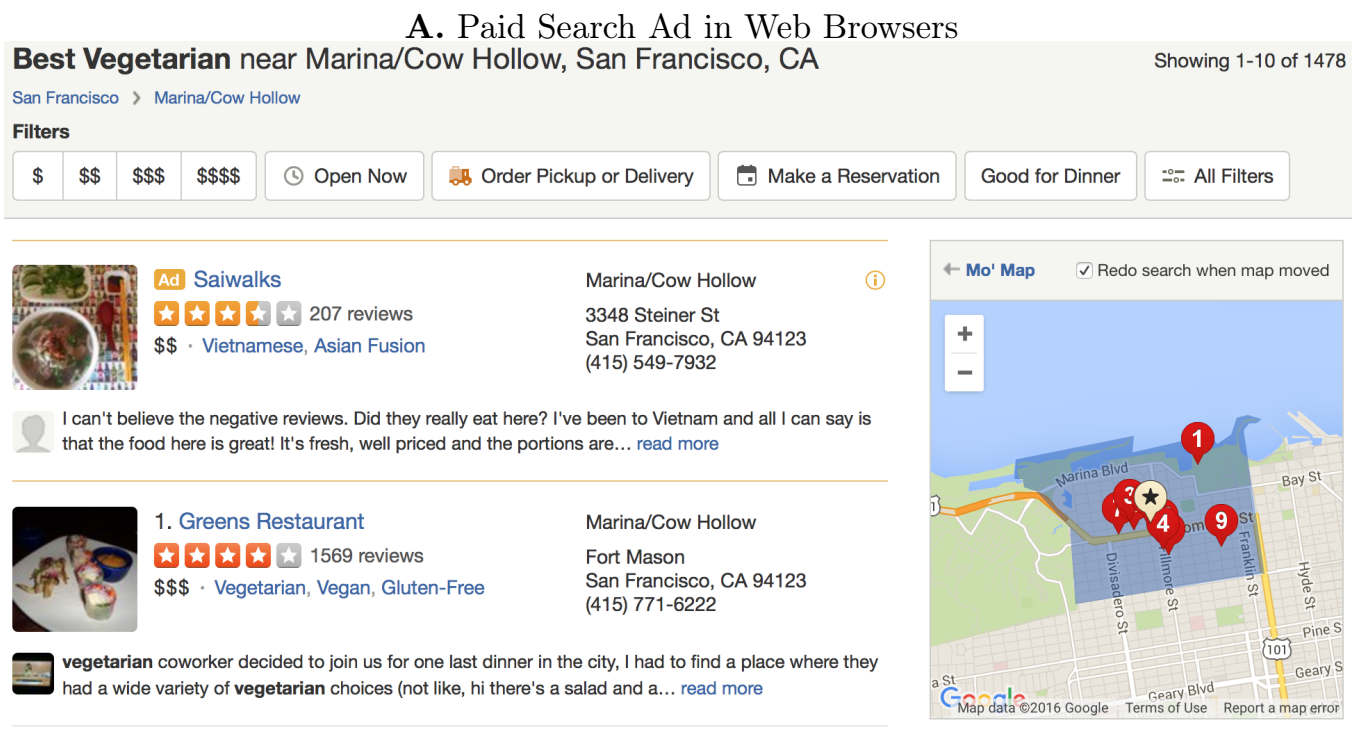

B. Paid Search Ad in Mobile App

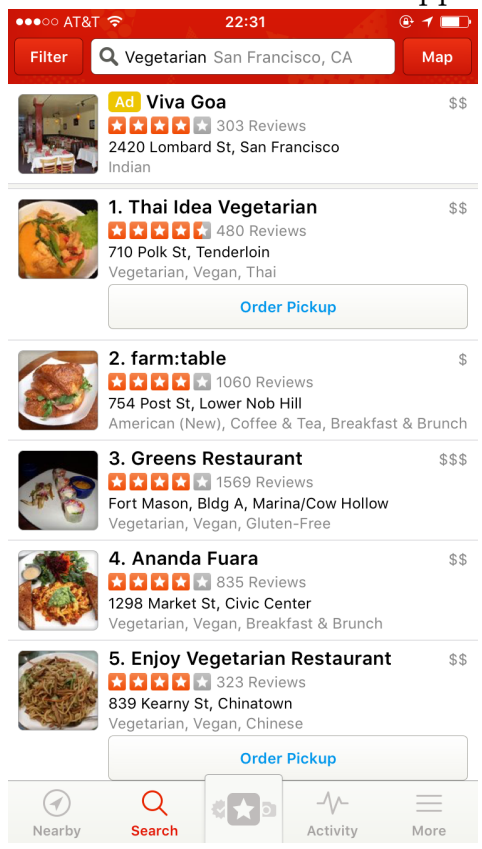

C.Paid Search Ad in a Mobile App Map

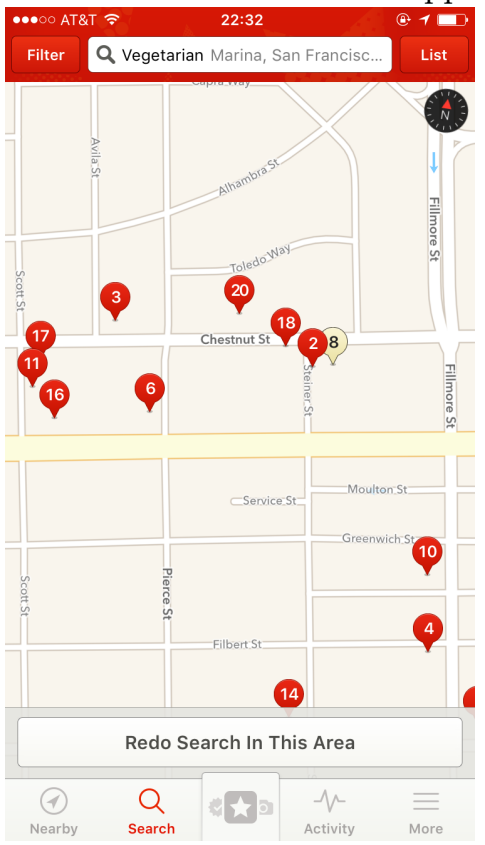

Notes: 1 The above figures are snapshots following a search session for "Vegetarian" near Marina, San Francisco, CA. The advertised restaurant is showed on the top of the page, marked by "Ad." The advertised restaurant is highlighted in yellow in the map, distinguished from other restaurants. 2 Also note that the search engine allows consumers to easily filter or identify restaurants that offer reservations through Yelp (Yelp Reservations restaurants) and restaurants that take delivery orders through Yelp (EAT24 restaurants). 


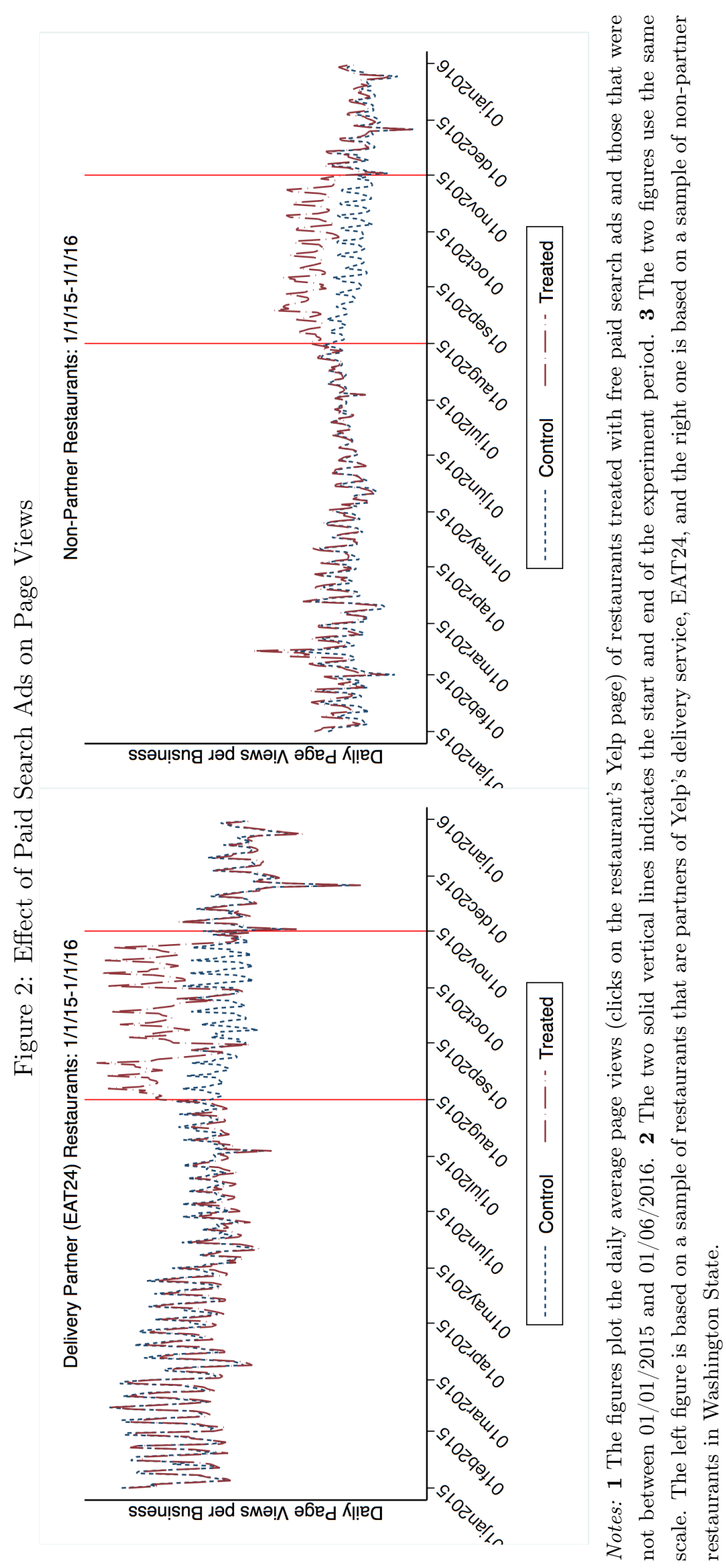


Figure 3: Histogram of Restaurant Ratings Before the Experiment

\section{A. Histogram of Restaurant Posted Ratings}
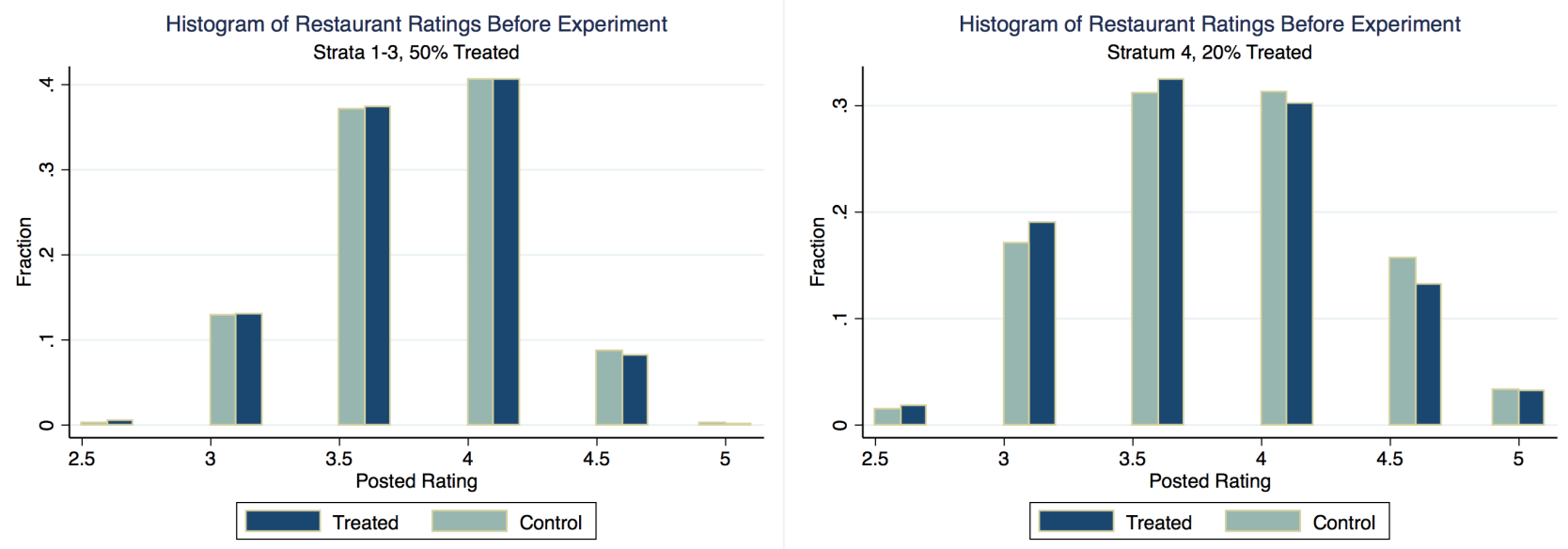

B. Histogram of Number of Reviews on Each Restaurant
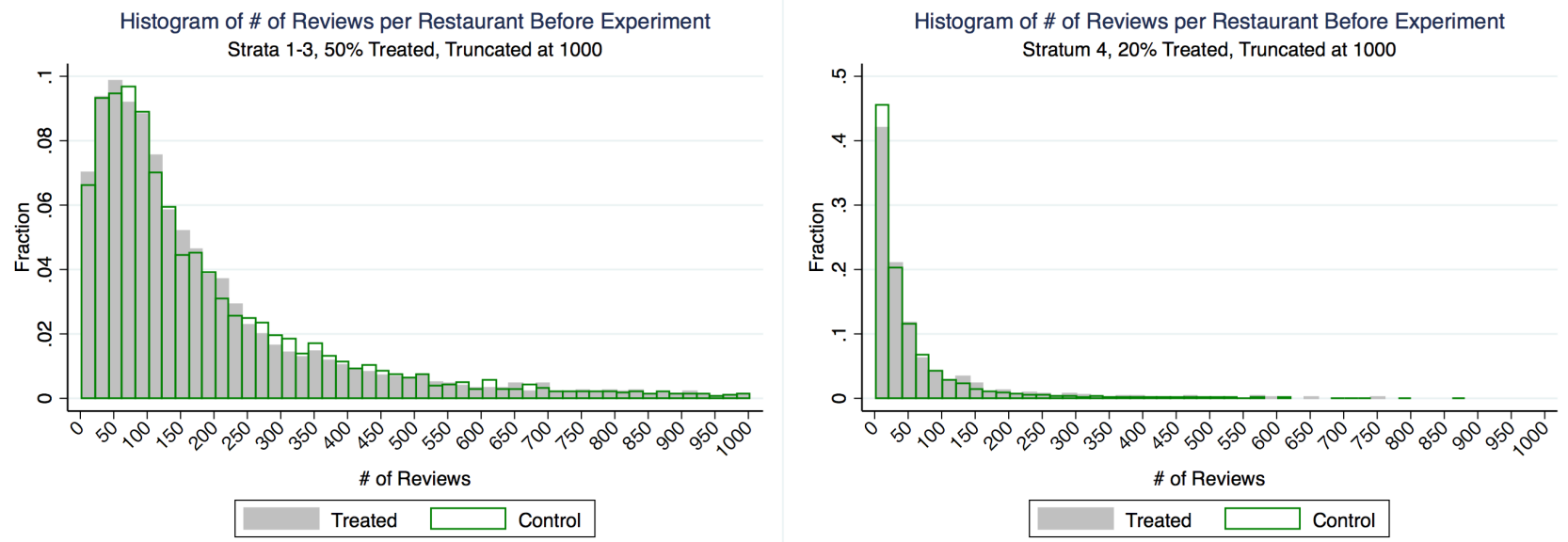

(Stratum 4 plotted separately due to different treatment sampling weight)

Notes: 1 Figure A plots the distribution of posted ratings by treatment status before the experiment. Figure B plots the distribution of the number of reviews by treatment status before the experiment. The distribution is truncated at 1,000 because of the extremely long right tail of the distribution. 2 We plot stratum 4 separately because only $20 \%$ are randomly assigned to be treated while $50 \%$ in other strata are treated. Two graphs need to be plotted separately to show balance in characteristics. The first three strata contain partners of Yelp Reservations (Yelp's reservation service), OpenTable, and EAT24 (Yelp's delivery service); the fourth stratum contains non-partner restaurants in Washington State. 3 We test characteristics balance between treated and control businesses within each stratum. The figures show that treated and control businesses have similar rating and number of reviews distributions. 
Figure 4: Average Effect of Paid Search Advertising

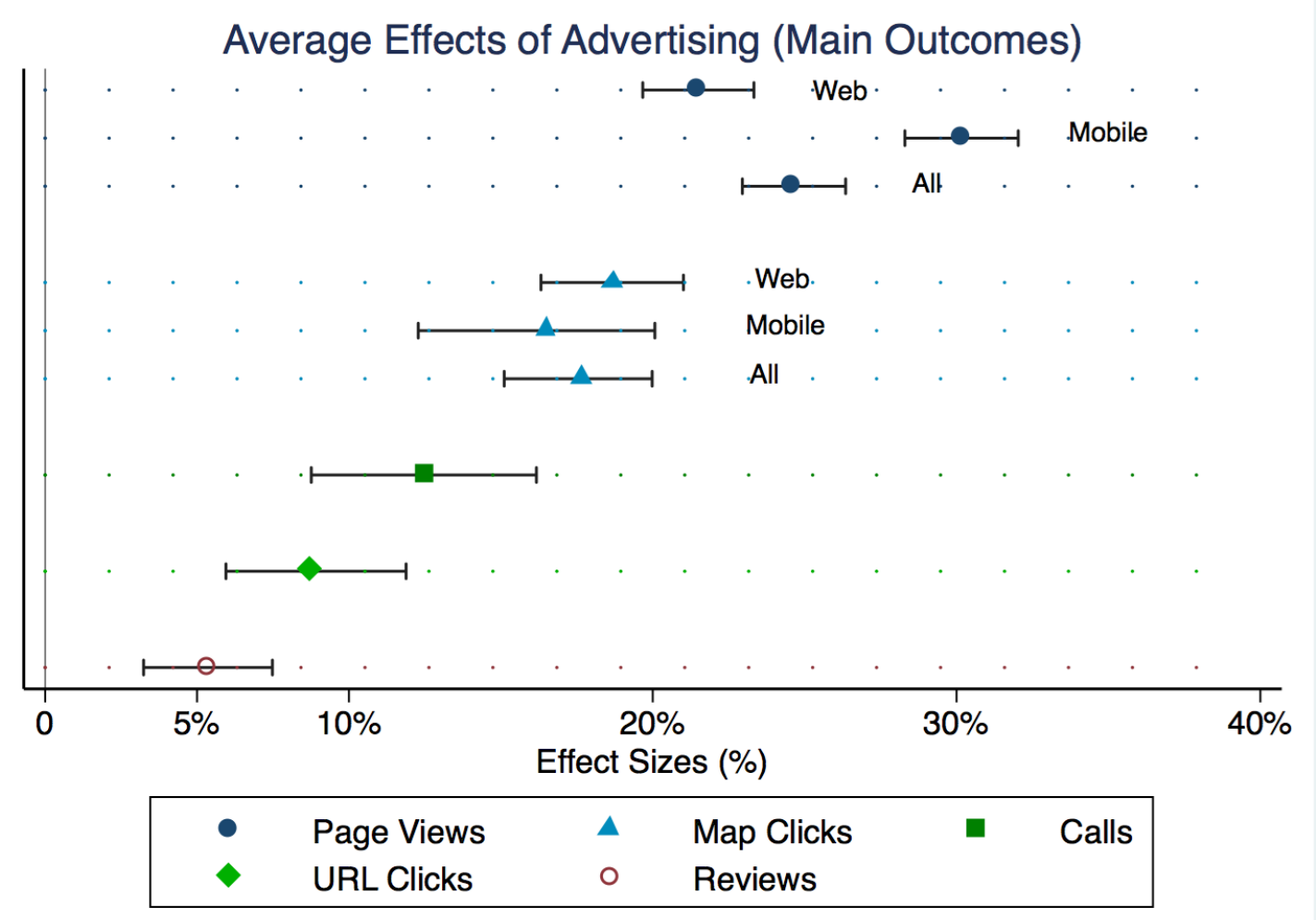

Notes: 1 The figure plots the average effects of paid search advertising and the confidence intervals of the estimates. 2 The estimates and confidence intervals are reported in Table 2. 3 The outcomes are page views (number of clicks on the restaurant's Yelp page), map clicks (number of times of the restaurant's map is queried), calls made to the restaurant through a mobile phone, clicks on the restaurant's URL link, and number of trusted reviews. 
Table 1: Experiment Sample Strata

\begin{tabular}{llccc}
\hline Block & Description & Sample & Treated & Control \\
Yelp Reservations & $\begin{array}{l}\text { Restaurants partnered with Yelp's } \\
\text { reservation service. }\end{array}$ & 307 & $50 \%$ & $50 \%$ \\
OpenTable & $\begin{array}{l}\text { Restaurants partnered with } \\
\text { OpenTable reservation service. }\end{array}$ & 5,740 & $49 \%$ & $51 \%$ \\
EAT24 & $\begin{array}{l}\text { Restaurants partnered with Yelp's } \\
\text { delivery service. }\end{array}$ & 5,867 & $50 \%$ & $50 \%$ \\
Others & Non-partners in Washington State & 6,381 & $20 \%$ & $80 \%$ \\
\hline & Total: & 18,295 & 7,210 & 11,085 \\
\hline
\end{tabular}


Table 2: Average Effects of Paid Search Advertising [95\% confidence interval]

\begin{tabular}{|c|c|c|}
\hline & Experiment-Period & Post-experiment-period \\
\hline \multicolumn{3}{|l|}{ Page Views } \\
\hline \multirow[t]{2}{*}{ on Web } & $21.5 \%$ & $0.8 \%$ \\
\hline & {$[19.7 \%, 23.3 \%]$} & {$[-1.3 \%, 2.9 \%]$} \\
\hline \multirow[t]{2}{*}{ on Mobile } & $30.2 \%$ & $1.9 \%$ \\
\hline & {$[28.3 \%, 32.0 \%]$} & {$[-0.3 \%, 4.1 \%]$} \\
\hline \multirow[t]{2}{*}{ Total } & $24.6 \%$ & $1.2 \%$ \\
\hline & {$[22.9 \%, 26.4 \%]$} & {$[-0.8 \%, 3.2 \%]$} \\
\hline \multicolumn{3}{|l|}{ Map Inquiries } \\
\hline \multirow[t]{2}{*}{$\overline{\text { on Web }}$} & $18.7 \%$ & $2.2 \%$ \\
\hline & {$[16.3 \%, 21.0 \%]$} & {$[-0.8 \%, 5.0 \%]$} \\
\hline \multirow[t]{2}{*}{ on Mobile } & $16.5 \%$ & $3.6 \%$ \\
\hline & {$[12.3 \%, 20.1 \%]$} & {$[0.9 \%, 6.1 \%]$} \\
\hline \multirow[t]{2}{*}{ Total } & $17.7 \%$ & $2.8 \%$ \\
\hline & {$[15.1 \%, 20.0 \%]$} & {$[0.2 \%, 5.2 \%]$} \\
\hline \multicolumn{3}{|l|}{ Other Conversions } \\
\hline \multirow[t]{2}{*}{ BusinessURL Clicks } & $8.8 \%$ & $-0.7 \%$ \\
\hline & {$[5.9 \%, 11.9 \%]$} & {$[-4.3 \%, 2.5 \%]$} \\
\hline \multirow[t]{2}{*}{ Calls to Businesses } & $12.6 \%$ & $0.6 \%$ \\
\hline & {$[8.8 \%, 16.2 \%]$} & {$[-3.1 \%, 4.1 \%]$} \\
\hline \multicolumn{3}{|l|}{ Check-Ins \& Reviews } \\
\hline \multirow[t]{2}{*}{$\begin{array}{l}\text { Check-Ins } \\
\text { Ch }\end{array}$} & $1.0 \%$ & $1.7 \%$ \\
\hline & {$[-1.0 \%, 3.1 \%]$} & {$[-1.7 \%, 5.0 \%]$} \\
\hline \multirow[t]{2}{*}{ \# of Trusted Reviews } & $5.4 \%$ & $1.5 \%$ \\
\hline & {$[3.2 \%, 7.5 \%]$} & {$[-1.5 \%, 4.2 \%]$} \\
\hline \multirow[t]{2}{*}{$\%$ of Trusted Reviews } & $-0.3 \%$ & $0.4 \%$ \\
\hline & {$[-1.2 \%, 0.4 \%]$} & {$[-0.6 \%, 1.3 \%]$} \\
\hline \multirow[t]{2}{*}{ Ratings } & $-0.3 \%$ & $0.2 \%$ \\
\hline & {$[-1.3 \%, 0.7 \%]$} & {$[-1.2 \%, 1.5 \%]$} \\
\hline \multirow[t]{2}{*}{ Review Length } & $-1.2 \%$ & $1.7 \%$ \\
\hline & {$[-3.2 \%, 0.8 \%]$} & {$[-0.9 \%, 4.2 \%]$} \\
\hline
\end{tabular}

95\% confidence interval in parentheses obtained from bootstrap.

Notes: 1 Table 2 reports the effects of advertising calculated by the method described in Section 3.2 The observations we obtained are between $1 / 1 / 2015$ and $12 / 31 / 2015$, and the experiment is conducted between $8 / 1 / 2015$ and $10 / 31 / 2015$. 